\title{
A Conceptual Model for Mobile Interaction using Brain Computer Interface
}

\author{
Abubaker Gaber ${ }^{1}$, Masitah Ghazali ${ }^{2}$ and Noorminshah A. Iahad ${ }^{3}$ \\ ${ }^{1}$ Dept. of Information Systems, Faculty of Computing, \\ Universiti Teknologi Malaysia \\ ${ }^{2}$ UTM ViCubeLab Research Group, Universiti Teknologi Malaysia \\ ${ }^{3}$ Dept. of Information Systems, Faculty of Computing, \\ Universiti Teknologi Malaysia \\ 1abujbr@gmail.com,2masitah@utm.my,3minshah@utm.my
}

\begin{abstract}
Interacting with mobiles and portable devices using brain waves has become a reality with the recent advances in the brain-computer interfaces (BCI) development. Today's BCI technology that provides portable headsets with wireless connectivity and supports multiple platforms, has paved path to many researches developing applications using those headsets on Android platforms. The common scenario however, although those BCI applications are installed on Android devices, the users can only interact with those dedicated BCI applications, but no other. In this study, a conceptual applications model has been proposed, as an alternative, for mobile interaction using BCI to allow a user to interact with operating system rather than interacting with only one dedicated application.
\end{abstract}

Keywords: Brain-computer interface, human-computer interaction, android, mobile interaction

\section{Introduction}

Brain is the center command system for the body [1] and it is filled with neurons. Every time we think, feel, move, or remember something, our neurons in our brains communicate with each other by sending small electric signals. Although the paths the signals take are insulated, some of the electric signal escapes, where the scientists can detect those signals [2]. A brain computer interface is a computer based system that acquires brain signals, analyses them, and translates them into commands that are relayed to an output device to carry out a desired action [3].

Brain Computer Interface (BCI) or Brain Machine Interface (BMI) or sometimes known as Mind Machine Interface (MMI), all refer to the same technique which is reading human brain waves using sensors and convert it into computer commands, enabling them to communicate directly with machines like wheelchair or a computer, either to assist them to communicate or to interact with their surroundings [4, 5]. In 1929, Hans Berger made a decisive breakthrough with the development of electroencephalography (EEG) to enable the noninvasive recording of neuroelectrical signals from the human brain [6]. The first attempt to control brain signals on a neurophysiological basis was reported in 1968, and the first scientific report of volitional control of human brain oscillation was published by Kamiya in 1969 [6].

The term brain computer interface was first proposed by Jacques Vidal in 1973, when he presented a system that could translate EEG signals into computer control signals [5], [6]. One of the main goals of $\mathrm{BCI}$ is to provide communications ability to severely disabled people [4], but it went beyond that and invade entertainment industry like games as new

Received (August 9, 2017), Review Result (November 15, 2017), Accepted (November 26, 2017) 
way of human-computer interaction and provide a unique experience as a game controller [7]. Researchers try their best to use BCI systems outside of the lab to enable patients to use that system in their daily life. To do so, they try to use BCI with portable devices instead of computers to receive the brain signal to take advantage of the portable devices like the small size and the long battery life compared to PC, because of the advanced of the technology the CPU of those portable devices getting more and more powerful [8].

In the last decade, technological advances in brain-computer interfaces permit EEG acquisition by means of wireless, mobile, dry, wearable, and low-cost EEG headsets, with new potential daily-life applications. The advances in the BCI development and their support to multiple operating systems including mobile OS like iOS and Android by providing software development kit (SDK) to developers led to more researches in BCI usability as a new way to interact with computes and mobiles [9]. But the quality of the signal that comes from those dry EEG is low compared to the traditional wet EEG signals, so it is not suitable for critical applications that may lead to harm the user, but it can be used as communication medium to control PC or mobile or even play games [9].There is a lot of dry and semi dry EEG BCI product in the market with Bluetooth connectivity that supports mobile operating system [9], for example NeuroSky Mindset and ENOBIO 8 wireless EEG system by Neuroelectrics [10], but the most popular EEG BCI product in the market are EPOC by Emotive, which it consists of 14 sensors and Neurosky Mindwave consist of one sensor, where both are using dry EEG and it is faster to setup than the wet EEG systems [11].

The advances in BCI have paved path to many researches to develop mobile BCI types of applications, specifically on Android platform, though those applications are still limited regarding the scope of control. For instance, if the user starts using the BCI for entertainment application, the scope of control is limited to that application only and cannot switch to another application unless the user touches the device screen. While BCI as a research field is obscure in terms of its applicability and development, researchers are still struggling to establish an accepted level of control over mobile platforms, in terms of general functionalities that are available to users. The goal of this study is to further develop a model of interaction on mobile platform, by proposing enhancements based on the observed limitations in existing studies, with a goal to enable users to control functionalities on their phones by brain signal, in layman's term, by simply thinking.

\section{BCI System}

BCI consists of hardware and software parts, and like any communication or control system, it has input (brain activity from the user) and output (commands) phases that translate the brain signal to command which goes through three main phases; signal acquisition, signal processing and finally the output command BCI system Figure 1 [12].

2.1. Signal Acquisition: Signal acquisition is the first phase of the BCI system which is getting the brain signals using the hardware (the EEG sensors) and recorded it from the scalp or the surface of the brain or record any neuronal activity within the brain [13].

2.2. Signal Processing: The second phase of the BCI system is the software phase which processes the brain signal and converts it to a computerized signal that the machines can understand, and it has two phases: feature extraction and translation algorithm [13]. 


\section{BCI SYSTEM}

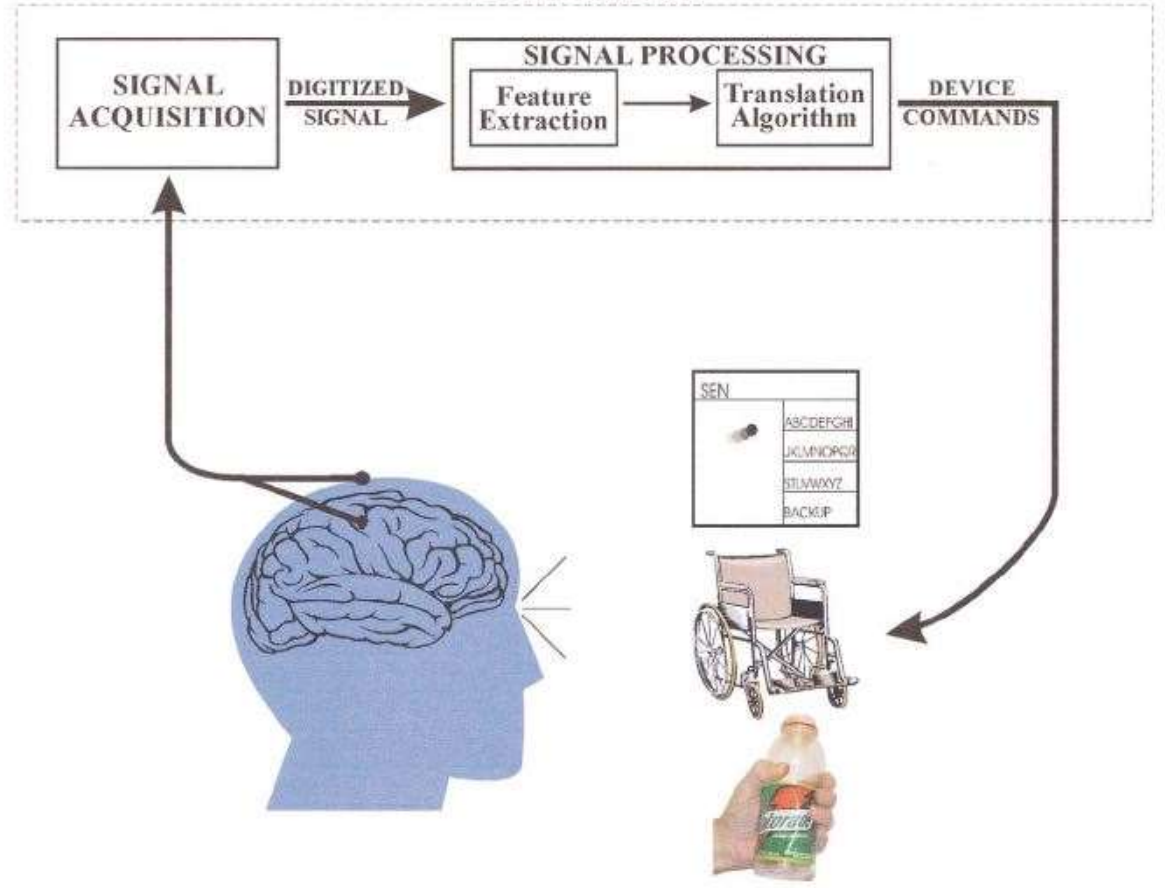

Figure 1. BCI System [12]

- Feature extraction: after getting the brain signals through the EEG sensors it goes to this phase which is analyzing the signal to extract the signal feature that encode the user's mental messages or commands. For example, visual evoked potential or Motor Imagery, the signal goes to the final phase of the process before it goes to the machine [13].

- Translation algorithm: the last phase of the signal processing is the translations algorithm, it takes the output of the feature extraction and translate the user's intent it to recognizable command by the output devices like machines and computers [13].

2.3. The Output Device: The output device is the final destination of the signal, the devices reward the user mental effort by responding to his mental command, for example cursor movement on computer screen or moving wheelchair [13].

\section{Tasks to Stimulate the Brain}

Brain-Computer Interface (BCI) monitors the user's brain activity and translates user intentions into commands to an external device, in order to generate brain activity, the user should do some mental task that also known as classification, for example movement imagination of right hand, left hand, or feet, this example called Motor imagery [14].

3.1. Motor Imagery: The user task is to imagine a movement of right hand, left hand, or feet, which then the EEG can read the brain signal and decode the user's intention, for example moving the cursor to the right or to the left [14].

3.2. Visual Evoked Potential: Visual evoked potential (VEP) depends on visual stimulation where the user is focusing on their target on the screen while the screen is flashing, the user's brain generates signals in respond to that visual stimulation, then the 
$\mathrm{BCI}$ detects those signals from the user brain and sends it as output command to the output device [15].

3.3. Blinking: Eyes blinking causes some changes in the brain signals of the user. The changes can be detected by the EEG sensors by comparing the brain signal while eyes are open and after the eye blink revealing the intentional eye blink. When user intentionally uses eye lids muscles to squeeze eyes, the BCI can detect soft and hard eye blinking based on squeezing power used by eye lid muscles and use it as command [16].

3.4. Attention and Meditation: Attention and meditation or in other word focus and relax has effect on the user brain. That mental stress can be detected by BCI and can be used by varies ways as command to control applications for communication purposes or for interments games [17].

\section{BCI Usage}

The immediate goal of BCI research is to provide communications capabilities to severely disabled people [4], by using brain signals to drive external devices without participation of the spinal and peripheral motor system, or for Yes and No communication, or even spelling a message [18]. There are two ways of using BCI in medical either as assistive BCIs to enable paralyzed patients to communicate or control external devices, or as rehabilitative BCIs to facilitate neural recovery [6]. Most of the applications have been developed for computers, but with current EEG wearable headsets which support Android development, has shifted from computers toward Android platforms.

\subsection{Communication}

People with severe physical disabilities could communicate again using BCI system and it gave them a good quality of life by providing them with a communication channel with their friends and family [19]. Simple way of communication provides people with severe physical disabilities with the ability to say yes or no, using their brain waves [19] or even using their eyes lid muscle to blink soft or hard, and that will give different output and use as communication channel [16].

These days, there are application like P300 speller based on visual evoked potential technique that helps people to select a character from the screen just by looking at their target on the screen, they can type and compose texts using BCI [20] and can send emails using this technique. BCI opens a new way for the people with severe physical disabilities [21] to access to the Internet, which could be a life changing for them as the Internet provides capabilities for education, communication, and even employment [19]. Communication application has been introduced to Android platform in terms of text editor in [22] by stimulating the user brain visually using Visual Evoked Potential technique to allow the user to compose a text message by selecting the desired letter from all the alphabets just by looking at a flashing letters on the Android device screen.

\subsection{Control}

Another important challenge for people with severe physical disabilities is controlling devices in the environment, for example, a television and make a phone call or moving a wheelchair. Recent work with BCI shows that it is possible, even to control robots with brain signals. Applications for neutrally controlled robots is currently centering on assistive technologies or in other words a helper robot, but BCI control has been proposed for military and industrial applications [19]. 
Early work in mobility employed small mobile robots is to simulate wheelchair navigation tasks with mental task based on BCI that operate a mobile robot to navigate in a home environment. The BCI research community's most effort is to develop methods for controlling mobility applications, like driving wheelchairs or manipulating remote robots [19]. Smart home nowadays is the perfect living environment for patients, where everything can be controlled remotely. For instance, in Jzau-Sheng Lin and Cheng-Hung Hsieh [23] experiment, they proposed in their paper about smart living system that can be controlled using BCI, like light, aircon, bed, TV and other home appliances using android platform. Another example of Android application that provide control ability to the user was presented in [24], where the user has the ability to control a wheelchair using and Android device to send the signal from BCI to Arduino device that control the wheelchair.

\subsection{Entertainment}

BCI systems went beyond medical applications to the entertainment industry like games, where the researchers found out that using BCI as a game controller could offer a new level of control possibilities for healthy users as well as people with severe physical disabilities [25]. It provides them with unique experience even simple games like Snake can be exciting while using BCI as controller [7]. In other example, before the paralyzed patients use BCI in the real environment, they have to be trained in a virtual reality, where researchers created a virtual environment for the paralyzed patients to be trained in [26].

Composing melody also become a reality for severely disabled people by using brain computer technology, where users can compose their melodies by picking the tune notes from the screen using BCI [27]. Brain computer interface has been used for entertainment games under Android platform in a racing game [17] where it was developed for Android and controlled using BCI.

\section{Limitation}

Based on our knowledge, up to this point, there is yet a fully interactive way to interact or control applications on Android operating system using BCI. Most of the Android applications that use BCI as interaction, or way of controlling the application, are limited in terms of the scope, i.e. the BCI can only interact with those dedicated applications, in [17] the user can only control the car inside the game. In simpler words, the user can only access or control that dedicated applications using BCI, and cannot switch to another type of application without having to touch the device screen. In addition, the user also cannot access or use the device functions that are not developed to interact with BCI.

\section{Conceptual Interaction Model}

Applications that use BCI to interact with, can be categorized to three main categories based on the usage (communication, entertainment and control). Most applications provide one of those types and it does not give the user the ability to switch or change the application type.

With this in mind, in the proposed conceptual model, the control application will be placed as the first type of application that should be provided to the user. The control application also appears as the output, as it should receive the BCI signal and simulate the main functions of the operating system like swapping and touching. Most control applications discussed previously provide the user with the ability to control a wheelchair or robotic hand. In this proposed conceptual model, the control application should give the user the ability to control the main functions of any operating system, that will provide the 
user with multiple applications and functionalities based on the operating system capability and on the user desire.

After providing the user with total control over the operating system, all other types of applications can be provided to the user, like communication applications and entertainment applications. Figure 2 is the proposed conceptual interacting model and it illustrates the application relation with operating system attached to the BCI system architecture that was introduced by Wolpaw [12] in Figure 1.

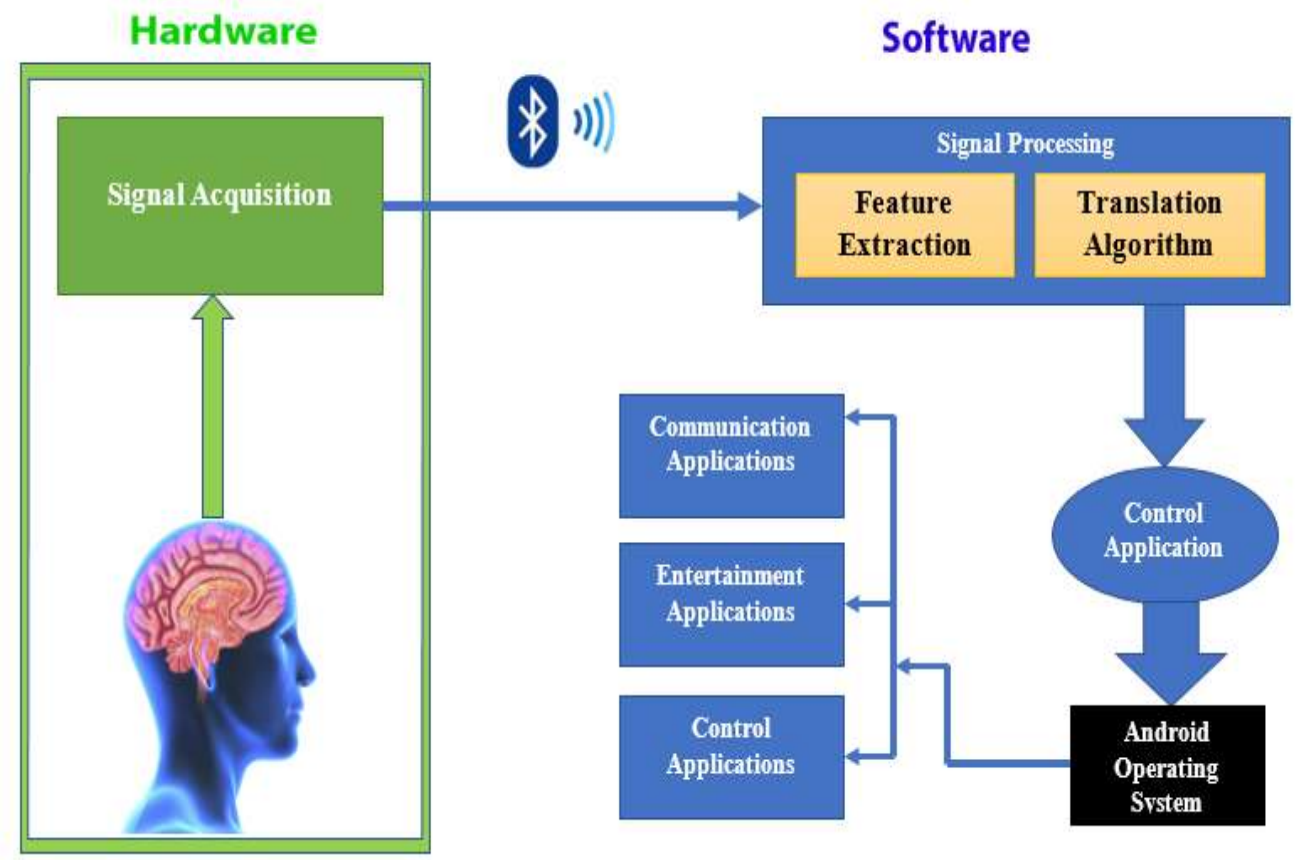

Figure 2. BCI Interaction Model

\section{Conclusion and Future Work}

Brain computer interface (BCI) is considered as an excellent communication channel for disabled people, to provide them with communication ability, entertainment and to control other devices as well. Rapid development of BCI led to provide portable headsets with Bluetooth connectivity and support for Android platforms, in which by using Android devices adds major value to the disabled people in terms of mobility and make their life better and easier.

Most of the current Android applications are dedicated applications, in which when these are used with BCI as interacting device, the users are somewhat limited to only using and manipulating the application they are currently accessing. Due to this reason, the users do not have the liberty to access to other functions and features of Android devices, apart from the application under used.

The proposed conceptual model aims to provide the user with full control over the operating system of an Android device. Besides having control over the main functions, such swapping left and right and simulate touch screen features without actually having to touch the screen, it also provides the user the freedom of choosing the desired application, desired content and accessing most, if not all, of the functions and features available in Android devices. As part of our future work, we plan to perform a usability study to further investigate the context and domain of when this interaction model would be deemed most effective and efficient. 


\section{Acknowledgment}

The authors would like to thank Universiti Teknologi Malaysia for providing financial support for this research under Research University Grant (Vote number: 18H32).

\section{References}

[1] E. E. Pastorino and S. M. Doyle-Portillo, What is Psychology? Cengage Learning, (2011).

[2] N. M. El-Kafrawy, D. Hegazy, and M. F. Tolba, "Features Extraction and Classification of EEG Signals Using Empirical Mode Decomposition and Support Vector Machine", in Advanced Machine Learning Technologies and Applications: Second International Conference, AMLTA 2014, Cairo, Egypt, November 28-30, 2014. Proceedings, A. E. Hassanien, M. F. Tolba, and A. Taher Azar, Eds. Cham: Springer International Publishing, (2014), pp. 189-198.

[3] S. Siuly, Y. Li, and Y. Zhang, "EEG Signal Analysis and Classification", no. 22306087, Cham: Springer International Publishing, (2016).

[4] L. F. Nicolas-Alonso and J. Gomez-Gil, "Brain computer interfaces, a review", Sensors (Basel), vol. 12, no. 2, (2012) January, pp. 1211-79.

[5] H. S. Anupama, N. K. Cauvery and G. M. Lingaraju, "Brain Computer Interface and Its Types - A Study", Int. J. Adv. Eng. Technol., vol. 3, no. 2, (2012), pp. 739-745.

[6] U. Chaudhary, N. Birbaumer and A. Ramos-Murguialday, "Brain-computer interfaces for communication and rehabilitation", Nat. Rev. Neurol., vol. 12, no. 9, (2016) August, pp. 513-525.

[7] A. I. Wang and E. A. Larsen, "Using Brain-Computer Interfaces in an Interactive Multimedia Application," in Parallel and Distributed Computing and Systems / 790: Software Engineering and Applications, (2012).

[8] G. Edlinger and C. Guger, "Laboratory PC and Mobile Pocket PC Brain-Computer Interface Architectures", Conf. Proc. ... Annu. Int. Conf. IEEE Eng. Med. Biol. Soc. IEEE Eng. Med. Biol. Soc. Annu. Conf., vol. 5, (2005), pp. 5347-50.

[9] J. Minguillon, M. A. Lopez-Gordo and F. Pelayo, "Trends in EEG-BCI for daily-life: Requirements for artifact removal”, Biomed. Signal Process, Control, vol. 31, (2017) January, pp. 407-418.

[10] M. Amlie, A. Kasim, C. Yee, M. Azmi and N. Ayuni, "ScienceDirect User-Friendly LabVIEW GUI for Prosthetic Hand Control using Emotiv EEG Headset”, Procedia - Procedia Comput. Sci., vol. 0, no. (2016) December, pp. 17-20.

[11] F. Lotte, L. Bougrain and M. Clerc, "Electroencephalography (EEG)-Based Brain-Computer Interfaces", in Wiley Encyclopedia of Electrical and Electronics Engineering, Hoboken, NJ, USA: John Wiley \& Sons, Inc., 2015, pp. 1-20.

[12] J. R. Wolpaw, N. Birbaumer, D. J. McFarland, G. Pfurtscheller and T. M. Vaughan, "Brain-computer interfaces for communication and control”, Clin. Neurophysiol., vol. 113, no. 6, (2002) June, pp. 767-91.

[13] J. R. Wolpaw, N. Birbaumer, D. J. McFarland, G. Pfurtscheller and T. M. Vaughan, "Brain-computer interfaces for communication and control", Clin. Neurophysiol., vol. 113, no. 6, (2002) June, pp. 767-91.

[14] S. Saeedi, R. Chavarriaga, I. Iturrate, J. d. R. Millan and T. Carlson, "Prediction of command delivery time for BCI", 2014 IEEE International Conference on Systems, Man, and Cybernetics (SMC), vol. 39, no. 3, (2014), pp. 1493-1498.

[15] G. Bin, X. Gao, Y. Wang, B. Hong and S. Gao, "VEP-based brain-computer interfaces: time, frequency, and code modulations [Research Frontier", IEEE Comput. Intell. Mag., vol. 4, no. 4, (2009) November, pp. 22-26.

[16] A. K. Singh, Y.-K. Wang, J.-T. King, C.-T. Lin and L.-W. Ko, "A simple communication system based on Brain Computer Interface", 2015 Conference on Technologies and Applications of Artificial Intelligence (TAAI), no. 1, (2015), pp. 363-366.

[17] G. Wu, Z. Xie and X. Wang, "Development of a mind-controlled Android racing game using a brain computer interface (BCI)", 2014 4th IEEE International Conference on Information Science and Technology, (2014), pp. 652-655.

[18] N. Birbaumer, A. R. Murguialday and L. Cohen, "Brain-computer interface in paralysis", Curr. Opin. Neurol., vol. 21, no. 6, (2008) December, pp. 634-638.

[19] M. Moore Jackson and R. Mappus, "Applications for Brain-Computer Interfaces", in Antimicrobial agents and chemotherapy, vol. 58, no. 12, (2010), pp. 89-103.

[20] M. Duvinage, "A P300-based Quantitative Comparison between the Emotiv Epoc Headset and a Medical EEG Device", in Biomedical Engineering / 765: Telehealth / 766: Assistive Technologies, (2012), pp. 37-42.

[21] S. Gannouni, N. Alrayes, A. A. A. Alsaudi and A. Alabdulkareem, "B-mail: A brain-controlled mail client," J. Digit. Inf. Manag., vol. 9, no. 4, (2011), pp. 167-170.

[22] A. S. Elsawy, S. Eldawlatly, M. Taher and G. M. Aly, "MindEdit: A P300-based text editor for mobile devices", Comput. Biol. Med., vol. 80, no. August 2016, (2017) January, pp. 97-106.

[23] J.-S. Lin and C.-H. Hsieh, "A Wireless BCI-Controlled Integration System in Smart Living Space for Patients”, Wirel. Pers. Commun., vol. 88, no. 2, (2016) May, pp. 395-412. 
[24] N. Shinde and K. George, "Brain-controlled driving aid for electric wheelchairs", 2016 IEEE 13th International Conference on Wearable and Implantable Body Sensor Networks (BSN), (2016), pp. 115118.

[25] P. Kierkegaard, "The brain as game controller”, Int. J. Liabil. Sci. Enq., vol. 3, no. 1-2, (2010), pp. $165-$ 77.

[26] F. Velasco-Alvarez, R. Ron-Angevin and M. A. Lopez-Gordo, "BCI-Based Navigation in Virtual and Real Environments", in Lncs, vol. 7903, (2013), pp. 404-412.

[27] B. Hamadicharef, M. Xu and S. Aditya, "Brain-Computer Interface (BCI) Based Musical Composition", in 2010 International Conference on Cyberworlds, (2010), pp. 282-286.

\section{Authors}

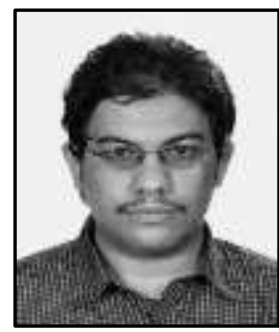

Abubaker Gaber, a Master student at Universiti Teknologi Malaysia in Information Technology Management, obtained his Bachelor of Computer Science in IT from Sikkim Manipal University, India.

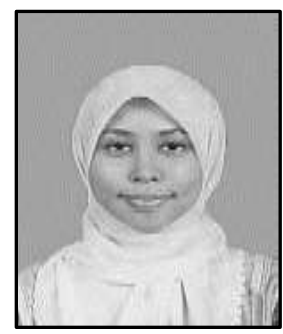

Masitah Ghazali, a senior lecturer at Software Engineering Department, Universiti Teknologi Malaysia and a member of UTM ViCubeLab research group. Awarded with Bachelor of Software Engineering from University of Manchester Institute of Science and Technology (UMIST), Master and PhD of Computer Science from Lancaster University, England.

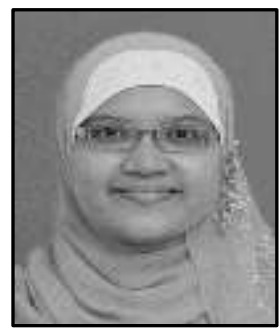

Noorminshah A. Iahad, a senior lecturer at Information Systems (IS) Department, Faculty of Computing, Universiti Teknologi Malaysia (UTM) and currently is an IT Manager at Research Management Center UTM. She was graduated with Bachelor of Science in IT at Universiti Malaya, Master of Computer Science in IS Engineering at UMIST, Manchester and her $\mathrm{PhD}$ in Computation at University of Manchester, England. 